\title{
Algebraic Analysis of Huzita's Origami Operations and their Extensions
}

\author{
Fadoua Ghourabi ${ }^{1}$, Asem Kasem ${ }^{2}$, and Cezary Kaliszyk ${ }^{3}$ \\ 1 University of Tsukuba, Japan \\ ghourabi@score.cs.tsukuba.ac.jp \\ 2 Yarmouk Private University, Syria \\ a-kasem@ypu.edu.sy \\ 3 University of Innsbruck, Austria \\ cezary.kaliszyk@uibk.ac.at
}

\begin{abstract}
We investigate the basic fold operations, often referred to as Huzita's axioms, which represent the standard seven operations used commonly in computational origami. We reformulate the operations by giving them precise conditions that eliminate the degenerate and incident cases. We prove that the reformulated ones yield a finite number of fold lines. Furthermore, we show how the incident cases reduce certain operations to simpler ones. We present an alternative single operation based on one of the operations without side conditions. We show how each of the reformulated operations can be realized by the alternative one. It is known that cubic equations can be solved using origami folding. We study the extension of origami by introducing fold operations that involve conic sections. We show that the new extended set of fold operations generates polynomial equations of degree up to six.
\end{abstract}

Keywords: fold operations, computational origami, conic section

\section{Introduction}

Origami is commonly conceived to be an art of paper folding by hand. It is not restricted to an art, however. We see continuous surge of interests in scientific and technological aspects of origami. It can be a basis for the study of geomet5 rical systems based on reflections, and the industrial applications can be found abundantly, such as in automobile industry, space industry etc.

In this paper, we focus on the algebraic and geometrical aspects of origami. Suppose that we want to construct a geometrically interesting shape, say a regular heptagon, from a sheet of paper. We need a certain level of precision even though we make the shape by hand. Since we do not use a ruler to measure the distance, nor do we use additional tools ${ }^{4}$, what we will do by hand is to construct creases and points. The creases are constructed by folding. The creases we make by folding are the segments of the lines that we will treat. The creases and the

\footnotetext{
${ }^{4}$ This restriction will be relaxed in Section 7 .
} 
four edges of the initial origami form line segments. The points are constructed 15 by the intersection of those segments. In the treatment of origami ${ }^{5}$ in this paper, origami is always sufficiently large such that, whenever we consider the intersections of segments, we consider the intersections of the lines that extend the segments. The shape of the origami that we want to obtain by folding is a (possibly overlaid) face(s) constructed by the convex set of the thus-constructed points.

As the crease is constructed by folding, the main question is how to specify the fold. Since we fold an origami along the line, the question is boiled down to how to specify the line along which we fold the origami. We call this line fold line.

In 1989 Huzita proposed the set of basic fold operations often referred to as Huzita's axiom set [4]. Later studies showed that Huzita's set of fold operations is more powerful than Euclidean tools, i.e. straightedge and compass (abbreviated to SEC hereafter), in that we can construct a larger set of points of coincidence by applying Huzita's set of operations than by SEC [1]. More precisely, the field of origami constructible numbers includes the field of SEC constructible numbers, therefore, the class of the shapes formed by connecting the coincidences is richer than that of the shapes formed by SEC. The trisector of a given arbitrary angle is a famous example that is constructible by origami, but not by SEC $[11,6]$. This triggered the activities of researchers who are interested in mathematical aspects 35 of origami such as the contribution of Martin [10] and Alperin [1]. Although several studies have been made to confirm the power of origami as we have seen above, we propose a more rigorous treatment of Huzita's set of operations. We choose not to use of terminology of Huzita's axiom itself as the set does not constitute a mathematical axiom set. The need for a formal method that can serve as a rigorous standard for the origami theory is pressing since folding techniques have been adapted in industry. This paper presents a preparatory but necessary rigorous statements of Huzita's basic fold operations towards the formalization of origami theory in proof assistants.

In this paper we restate Huzita's basic fold operations. We make the new statements more precise by clarifying the conditions that enable folds. We analyze the operations algebraically and present theorems about the finite number of fold lines. We also introduce a general origami principle that performs all the operations. Furthermore, we extend the capability of basic fold operations by introducing conic sections and show that this extension is defined by equations of degree six.

The structure of the rest of the paper is as follows. In Section 2, we summarize our notions and notations. In Section 3, we present Huzita's basic fold operations. In Section 4, we define the possible superpositions of geometrical objects of origami. In Section 5, we reformulate the basic fold operations. In Section 6, we ${ }_{55}$ introduce a general origami principle that performs all the basic fold operations.

\footnotetext{
5 The word origami is also used to refer to a square sheet of paper used to perform origami.
} 
In Section 7, we consider superpositions of points and conic sections. In Section 8, we conclude with remarks on future directions of research.

\section{Preliminaries}

An origami is notated by $\mathcal{O}$. An origami $\mathcal{O}$ is supposed to represent a square sheet of paper with four points on the corners and four edges that is subject to folding. Some intersections of lines may not fit on the square paper. However, we want to work with these points. To achieve this, we consider $\mathcal{O}$ to be a sufficiently large surface so that all the points and lines that we treat are on $\mathcal{O}$.

In this paper, we restrict the use of geometrical objects only to points, lines and s-pairs (to be defined in Section 4). We use $\alpha$ and $\beta$ to note either a point or a line. Points are notated by a single capital letter of the Latin alphabet such as $A, B, C, D, P, Q$ etc. $^{6}$, and lines are notated by $\gamma, k, m$, and $n$. Since we use Cartesian coordinate system in this paper, a line is represented by a linear equation $a x+b y+c=0$ in variables $x$ and $y$. The notation " $f(x, y):=$ polynomial in $x$ and $y=0$ " is used to declare that $f$ is a curve represented by the equation polynomial $=0 .(x, y)$ on the lefthand side of $:=$ may be omitted.

The sets of all points and lines are notated by $\Pi$ and $\mathcal{L}$, respectively. Abusing the set notation we use $P \in m$ to mean point $P$ is on line $m$.

For a set $\mathcal{S}$, we notate its cardinality by $|\mathcal{S}|$. For two lines $m$ and $n, m \| n$ is true when $m$ and $n$ are parallel or equal.

\section{Fold Principle}

\subsection{Basic idea}

By hand we can fold the origami by tentatively making a line either to let it pass through two points or to superpose points and lines. The former corresponds to applying a straightedge in Euclidean construction. In practice, to construct a line that passes through a point we bend the paper near the point and we flatten the paper gradually until we make the point lie on the intended fold line. The latter is pertinent to origami. Superposition involves two points, a point and a line, and two lines. To superpose two points, we bring one point to another, and then we

85 flatten the paper. To superpose a point and a line, the easy way is to bring the point onto the line, and then we flatten the paper. Superposition of two lines is more complex, and we will treat the operation along with its algebraic meaning in Section 4.

\subsection{Huzita's Basic Fold Operations}

We restate the set of seven basic fold operations of Huzita. The first six were proposed by Huzita and below are their statements as they appear in [4]. The

${ }^{6} A \sim F, X$ and $Y$ are overloaded, in fact. The meaning the symbols denote should be clear from the context. 
seventh was proposed by Justin [7] and rephrased by us to fit in with Huzita's statements.

(1) Given two distinct points, you can fold making the crease pass through both points (ruler operation).

(2) Given two distinct points, you can fold superposing one point onto the other point (perpendicular bisector).

(3) Given two distinct (straight) lines, you can fold superposing one line onto another (bisector of the angle).

(4) Given one line and one point, you can fold making the crease perpendicular to the line and passing through the point (perpendicular footing).

(5) Given one line and two distinct points not on this line, you can fold superposing one point onto the line and making the crease pass through the other point (tangent from a point to a parabola).

(6) Given two distinct points and two distinct lines, you can fold superposing the first point onto the first line and the second point onto the second line at the same time.

(7) Given a point and two lines, you can fold superposing the point onto the first line and making the crease perpendicular to the second line.

We will call this set of basic fold operations Huzita's fold principle.

The set of points and lines that can be constructed using the first five and seventh operations is the same, as the set of points and lines that can be constructed by SEC. The sixth operation is more powerful: it allows constructing common tangents to two parabolas which are not realizable by SEC.

Huzita and Justin carefully worked out the statements to exclude the cases that give infinite fold lines by imposing conditions on points and lines (e.g. distinct points, distinct lines, etc.). However, some of these conditions are insufficient or unnecessary. A thorough discussion on Huzita's statements is given in $[9]$.

${ }_{120}$ While these statements are suitable for practicing origami by hand, a machine needs stricter guidance. An algorithmic approach to folding requires formal definition of fold operations. Furthermore, we need to explicitly identify the conditions that ensure the finite number of fold lines.

\section{Superposition}

${ }_{125}$ We define a superposition pair, s-pair for short, $(\alpha, \beta)$. It is a pair of geometrical objects $\alpha$ and $\beta$ that are to be superposed. An s-pair $(\alpha, \beta)$ defines a fold line along which the origami is folded to superpose objects $\alpha$ and $\beta$. Depending upon the types of the objects, we have the following superpositions.

Point-point superposition When points $P$ and $Q$ are distinct, the s-pair $130 \quad(P, Q)$ defines a unique fold line that superposes $P$ and $Q$. This unique line is the perpendicular bisector of the line segment whose start and end points are $P$ and $Q$, respectively, and is denoted by $P \uparrow Q$ 
When points $P$ and $Q$ are equal, the s-pair $(P, Q)$, i.e. the s-pair $(P, P)$, does not define a unique fold line to superpose $P$ onto itself. Points $P$ and $P$ are superposed by any fold line that passes through $P$. Namely, the s-pair $(P, P)$ defines the infinite set $\mathcal{I}(P)$ of fold lines that pass through $P$, i.e.

$$
\mathcal{I}(P)=\{\gamma \mid P \in \gamma\}
$$

Here we note that two sets $\mathcal{I}(P)$ and $\mathcal{I}(Q)$ can define a line, denoted by $P Q$, passing through points $P$ and $Q$, i.e. $\gamma=\mathcal{I}(P) \cap \mathcal{I}(Q)$. The straightedge operation can even be replaced by the superpositions.

Line-line superposition When lines $m$ and $n$ are equal, what we do is superposing a line onto itself. This is achieved in the following way. Choose an arbitrary point on the line, divide the line into the two half lines, and then superpose the two half lines with the dividing point at the ends of the both half lines. Geometrically speaking, we construct a perpendicular to $m$ and fold along that perpendicular. Any perpendicular to $m$ superposes the line $m$ onto itself. Hence, the s-pair $(m, m)$ defines the following infinite set $\mathcal{B}(m)$ of fold lines.

$$
\mathcal{B}(m)=\{X \uparrow Y \mid X, Y \in m, X \neq Y\}
$$

Note that, in passing, we exclude $m$ itself from $\mathcal{B}(m)$. Namely, $m$ is not considered as the fold line to superpose $m$ onto itself as this does not create new lines.

150 To superpose two distinct lines, we assume the capability of hands that slides a point along a line. By the combination of superposition and of sliding, we can achieve the superposition of two distinct lines.

Point-line superposition An s-pair $(P, m)$ defines the following set $\Gamma(P, m)$ of fold lines that superpose $P$ and $m$.

$$
\Gamma(P, m)= \begin{cases}\{X \uparrow P \mid X \in m\} & \text { if } P \notin m \\ \mathcal{B}(m) \cup \mathcal{I}(P) & \text { if } P \in m\end{cases}
$$

Here we define $\Gamma(P, m)$ by cases of $P \notin m$ and $P \in m$. If $P \notin m$ then the fold line that superposes $P$ and $m$ is a tangent to the parabola with focus $P$ and directrix $m[7,10,1]$. Therefore, $\{X \uparrow P \mid X \in m\}$, in the former case, denotes the set of tangents of the parabola defined by the focus $P$ and the directrix $m$. The latter corresponds to folding along any perpendicular to $m$ or along any line that passes through $P$.

\section{Formulation of Fold}

\subsection{Revisit of Huzita's Fold Principle}

Table 1 shows the reformulation of Huzita's fold principle by a superposition or combinations of two superpositions. Each row of Table 1 corresponds to each 
basic operation given in Subsection 3.2. The second column shows the superpositions used to formalize each fold operation. The third column summarizes the degenerate cases of each operation. In practice, a degenerate case means infinite folding possibilities to achieve the superpositions in the second column. Huzita implicitly assumed $P \notin m$ whenever a point $P$ and a line $m$ are to be superposed. The fourth column indicates this assumption of the incidence relation. An incident case is one where the s-pair $(\alpha, \beta) \in \Pi \times \mathcal{L}$ has the property $\alpha \in \beta$. This can occur in the operations where we have point-line superposition(s), namely operations $(5),(6)$ and $(7)$. In the case of (6), it is enough to have only one s-pair that has the property $\alpha \in \beta$.

Propositions of incidence may cover some of degenerate configurations. In Table 2, redundancy of propositions is avoided in the last two columns. For instance in the case of (5), if $P=Q \wedge P \in m$ then there are infinite possible fold lines passing through $Q$ and superposing $P$ and $m$. More precisely, any line passing through $P$ is a possible fold line. Or, the proposition $P \in m$ of incidence covers the degeneracy proposition. In other words, by eliminating the case where $P \in m$, we also eliminate the case where $P=Q$ and $P \in m$. The proposition $P=Q \wedge P \in m$ is removed from degeneracy column of the operation (5) in Table 2. The more general condition, i.e. $P \in m$, is kept in the incidence column.

Table 1. Superpositions in Huzita's fold principle

\begin{tabular}{|c|c|c|c|}
\hline operation & s-pairs & degeneracy & incidence \\
\hline$(1)$ & $(P, P),(Q, Q)$ & $P=Q$ & - \\
\hline$(2)$ & $(P, Q)$ & $P=Q$ & - \\
\hline$(3)$ & $(m, n)$ & $m=n$ & - \\
\hline$(4)$ & $(m, m),(P, P)$ & - & - \\
\hline$(5)$ & $(P, m),(Q, Q)$ & $P=Q \wedge P \in m$ & $P \in m$ \\
\hline$(6)$ & $(P, m),(Q, n)$ & $\begin{array}{c}(P \in m \wedge Q \in n \wedge(m \| n \vee P=Q)) \vee \\
(P \notin m \wedge Q \notin n \wedge m=n \wedge P=Q)\end{array}$ & $P \in Q \in n$ \\
\hline$(7)$ & $(P, m),(n, n)$ & $m \| n \wedge P \in m$ & $P \in m$ \\
\hline
\end{tabular}

The notion of superposition enable us to reformulate Huzita's fold principle. We first introduce a function $\zeta$ that, given a sequence of s-pairs, computes all the fold lines that realize all the given s-pairs (i.e. superpose the elements). The detailed definition of $\zeta$ is beyond the scope of this paper. Function $\zeta$ has been implemented as the core of computational origami system Eos $[3,5]$. We provide the reformulation of Huzita's fold principle: a new set of operations that specify $\zeta$. We denote this new formalization by $\mathbf{H}$.

(O1) Given two distinct points $P$ and $Q$, fold $\mathcal{O}$ along the unique line that passes through $P$ and $Q$. 
Table 2. Superpositions in Huzita's fold principle with simpler conditions for degeneracy

\begin{tabular}{|c|c|c|c|}
\hline operation & s-pairs & degeneracy & incidence \\
\hline$(1)$ & $(P, P),(Q, Q)$ & $P=Q$ & - \\
\hline$(2)$ & $(P, Q)$ & $P=Q$ & - \\
\hline$(3)$ & $(m, n)$ & $m=n$ & - \\
\hline$(4)$ & $(m, m),(P, P)$ & - & - \\
\hline$(5)$ & $(P, m),(Q, Q)$ & - & $P \in m$ \\
\hline$(6)$ & $(P, m),(Q, n)$ & $P=Q \wedge m=n$ & $P \in m \vee Q \in n$ \\
\hline$(7)$ & $(P, m),(n, n)$ & - & $P \in m$ \\
\hline
\end{tabular}

(O2) Given two distinct points $P$ and $Q$, fold $\mathcal{O}$ along the unique line to superpose $P$ and $Q$.

(O3) Given two distinct lines $m$ and $n$, fold $\mathcal{O}$ along a line to superpose $m$ and $n$.

(O4) Given a line $m$ and a point $P$, fold $\mathcal{O}$ along the unique line passing through $P$ to superpose $m$ onto itself.

(O5) Given a line $m$, a point $P$ not on $m$ and a point $Q$, fold $\mathcal{O}$ along a line passing through $Q$ to superpose $P$ and $m$.

(O6) Given two lines $m$ and $n$, a point $P$ not on $m$ and a point $Q$ not on $n$, where $m$ and $n$ are distinct or $P$ and $Q$ are distinct, fold $\mathcal{O}$ along a line to superpose $P$ and $m$, and $Q$ and $n$.

(O7) Given two lines $m$ and $n$ and a point $P$ not on $m$, fold $\mathcal{O}$ along the unique line to superpose $P$ and $m$, and $n$ onto itself.

The above statements of $(\mathrm{O} 1) \sim(\mathrm{O} 7)$ include the conditions that eliminates degeneracy and incidence. These conditions correspond to the negations of the propositions of third and fourth column of Table 2 in natural language.

Using $\zeta$, we define origami constructible objects.

Definition 1 (Origami constructible objects). Given a set of initial objects $\mathcal{S}(\subseteq \Pi \cup \mathcal{L})$, the set of origami constructible objects is inductively defined as the least set containing origami constructible objects given in 1. $\sim 4 .:$

1. A point $P$ is origami constructible, if $P \in \mathcal{S}$.

2. An s-pair $(\alpha, \beta)$ is origami constructible if $\alpha$ and $\beta$ are origami constructible.

3. A line $\gamma$ is origami constructible if $\gamma \in \zeta(\underline{s})$ and $\underline{s}$ is a sequence of origami constructible s-pairs.

4. The intersection of lines $m$ and $n$ is origami constructible if $m$ and $n$ are origami constructible.

One may wonder why the reflection of an origami constructible point across an origami constructible line is not included in this definition. In fact, reflections are constructible using the operations of $\mathbf{H}$ [10]. In practice, however, reflections are treated as if they were in the above inductive definition. 


\subsection{Properties of Operations in $\mathbf{H}$}

We now study the properties of the operations in $\mathbf{H}$. For each operation, we will show the finiteness of the number of the constructible fold lines under certain conditions. This result is important to ensure the decidability of the fold since otherwise we would have an infinite computation. For each of $(\mathrm{O} 1),(\mathrm{O} 2)$ and (O4), we have a unique fold line.

Since all the objects that we study now are origami constructible, the sets of points and lines are now denoted by $\Pi_{\mathcal{O}}$ and $\mathcal{L}_{\mathcal{O}}$ (each subscripted by $\mathcal{O}$ ) in all the propositions to follow. The first two are easy ones.

Proposition 1 (Fold line of (O1)).

$$
\forall P, Q \in \Pi_{\mathcal{O}} \text { such that } P \neq Q, \quad \exists ! \gamma \in \mathcal{I}(P) \cap \mathcal{I}(Q) .
$$

This unique $\gamma$ is denoted by $P Q$.

Proposition 2 (Fold line of (O2)).

$\forall P, Q \in \Pi_{\mathcal{O}}$ such that $P \neq Q, \quad \exists ! \gamma=P \uparrow Q$.

Proposition 3 (Fold line of (O4)).

$$
\forall m \in \mathcal{L}_{\mathcal{O}} \forall P \in \Pi_{\mathcal{O}} \quad \exists ! \gamma \in \mathcal{B}(m) \cap \mathcal{I}(P)
$$

A fold line in $(\mathrm{O} 5)$ is determined by s-pairs $(P, m)$ and $(Q, Q)$ under the condition of $P \notin m$. The fold in (O5) is impossible in certain configurations. The following proposition more sharply describes this property.

Proposition 4 (Fold lines of (O5)).

$$
\forall m \in \mathcal{L}_{\mathcal{O}} \forall P, Q \in \Pi_{\mathcal{O}} \text { such that } P \notin m
$$

240

$$
|\Gamma(P, m) \cap \mathcal{I}(Q)| \leqslant 2 .
$$

Proof. The algebraic proof of this proposition is straightforward and extendable to the general cases of conic sections. Recall that $\Gamma(P, m)$ defines the set of the tangents of the parabolas whose focus and directrix are $P$ and $m$, respectively. A general form of an equation of the parabola is given by the following irreducible polynomial equation

$$
f(x, y):=A x^{2}+B x y+C y^{2}+D x+E y+F=0,
$$

where $A, B, C, D, E$ and $F$ are constants, not both $A$ and $B$ are 0 , and $B^{2}=$ $4 A C$. The tangent to the curve $f(x, y)$ at the point $(X, Y)$ is given by

$$
g(x, y):=\frac{\partial f}{\partial x}(X, Y) \cdot(x-X)+\frac{\partial f}{\partial y}(X, Y) \cdot(y-Y)=0 .
$$


Let $Q$ be $(u, v)$. As the line $g$ passes through $Q$, we have $g(u, v)=0$. We will solve for $X$ and $Y$ of the system of equations

$$
\{f(X, Y)=0, g(u, v)=0\} .
$$

Since $g(u, v)$ is linear in $X$ and $Y$, finding the solutions is reduced to solving, in $X$ (or in $Y$ ), the (at most) second degree polynomial equation obtained from $f(X, Y)=0$ by eliminating either $Y$ or $X$. Obviously, the number of real solutions is less or equal to 2 .

Concerning (O7), the following proposition holds.

Proposition 5 (Fold lines of (O7)).

$$
\begin{gathered}
\forall m, n \in \mathcal{L}_{\mathcal{O}} \forall P \in \Pi_{\mathcal{O}} \text { such that } P \notin m, \\
|\Gamma(P, m) \cap \mathcal{B}(n)| \leqslant 1 .
\end{gathered}
$$

Proof. The proof is similar to the proof of Proposition 4. We use the formula (5.1) there. Instead of the condition that the tangent passes through a particular point, we impose the condition that the slope of the tangent at point $(X, Y)$ is given, say $k(\neq \infty)$, in this proposition. From Eq. (5.1), we have the equation representing the tangent at $(X, Y)$.

$$
h(x, y):=D+2 A x+B y+(E+B x+2 C y) \frac{d y}{d x}(X, Y)=0
$$

Since $k\left(=\frac{d y}{d x}(X, Y)\right)$ is given, all we need is to solve for $X$ and $Y$ in the system of equations

$$
\{f(X, Y)=0, D+2 A X+B Y+(E+B X+2 C Y) k=0\}
$$

It is easy to see that we have at most two real solutions for the pair $(X, Y)$.

However, when $B^{2}=4 A C$, which is the case of the parabola, we have at most one real solution by an easy symbolic computation by computer algebra systems.

Most interesting case is (O6), which actually gives extra power over SEC.

\section{Proposition 6 (Fold lines of (O6)).}

$$
\begin{array}{r}
\forall m, n \in \mathcal{L}_{\mathcal{O}} \forall P, Q \in \Pi_{\mathcal{O}} \text { such that } P \notin m \wedge Q \notin n \\
\neg(P=Q \wedge m=n) \Rightarrow \\
\text { if } m \| n \text { then }|\Gamma(P, m) \cap \Gamma(Q, n)| \leqslant 2 \\
\text { else } 1 \leqslant|\Gamma(P, m) \cap \Gamma(Q, n)| \leqslant 3 .
\end{array}
$$


Proof. Instead of the general equation (5.1) of the conic section, we use the following equation for the parabola defined by the focus $(u, v)$ and the directrix $a x+b y+c=0$.

$$
f(x, y):=\left(a^{2}+b^{2}\right)\left((x-u)^{2}+(y-v)^{2}\right)-(a x+b y+c)^{2}=0 .
$$

265

We only have to consider the cases of $m \neq n$ and of $P \neq Q \wedge m=n$. We consider the former, first. Let $f_{i}(x, y)$ be the function given in (5.6) with all the constants $a, b, c, u$ and $v$ being indexed by $i$.

Let $P$ and $Q$ be points at $\left(u_{1}, v_{1}\right)$ and at $\left(u_{2}, v_{2}\right)$ respectively, and $m$ and $n$ be the line $a_{1} x+b_{1} y+c_{1}=0$, and $a_{2} x+b_{2} y+c_{2}=0$, respectively. Note that we can give a unique representation for the same line, so that the two lines are equal iff each coefficient $a, b$ and $c$ for each equation are equal. Now, let $f_{1}$ and $f_{2}$ be the parabolas defined by $P$ and $m$, and by $Q$ and $n$, respectively.

We distinguish the following two cases.

1. $m \nVdash n$

$275 \quad$ As in the proof of Proposition 5, we derive the the tangent $h_{1}$ with the slope $t$ at point $\left(X_{1}, Y_{1}\right)$ on $f_{1}(x, y)=0$, and the tangent $h_{2}$ with slope $t$ at point $\left(X_{2}, Y_{2}\right)$ on $f_{2}(x, y)=0$. The system $\left\{f_{1}\left(X_{1}, Y_{1}\right)=0, h_{1}\left(X_{1}, Y_{1}\right)=0\right\}$ yields $X_{1}$ and $Y_{1}$ as functions of $t$. Similarly, we obtain $X_{2}$ and $Y_{2}$ as functions of $t$. Since $\left(Y_{1}-Y_{2}\right)-t\left(X_{1}-X_{2}\right)=0$, we have the polynomial equation, whose polynomial is degree 3 in $t$. Hence, the number of distinct real solutions is 1,2 or 3 .

2. $m \| n$

Similarly to case 1 ., we obtain the polynomial equation of degree 2 in $t$. Hence we have 1 or 2 distinct real solutions.

What remains to be considered is the case of $P \neq Q \wedge m=n$. Similarly to the case 2, above, we obtain the polynomial equation of degree 2 in $t$. Furthermore, the discriminant of the obtained equation is easily shown to be non-negative. Hence, the relation (5.5) follows.

Operation (O3) is a special case of $(\mathrm{O} 6)$ with $m=n$ and $P \neq Q$. In this case, the fold operation is about superposing the two lines $P Q$ and $m$. As the corollary of Proposition 6.

Proposition 7 (Fold lines of (O3)).

$$
\begin{array}{r}
\forall m \in \mathcal{L}_{\mathcal{O}} \quad \forall P, Q \in \Pi_{\mathcal{O}} \quad P \neq Q \Rightarrow \\
1 \leqslant|\Gamma(P, m) \cap \Gamma(Q, m)| \leqslant 2 .
\end{array}
$$

\section{General Origami Principle}

Since the algebraic interpretation of (O6) can be expressed by a cubic equation, a natural question is whether $(\mathrm{O} 6)$ can do all the rest of fold operations of $\mathbf{H}$ with certain side conditions. The answer is basically, yes, but we need to carefully 
analyze the degenerate and incident cases, which will form the premise of the implicational formula of Lemma 1 that we will prove next.

We start with the general origami principle, which we denote by $\mathbf{G}$, that consists of the following single operation.

(G) Given two points $P$ and $Q$ and two lines $m$ and $n$, fold $\mathcal{O}$ along a line to superpose $P$ and $m$, and $Q$ and $n$.

Operation $(\mathrm{G})$ is obtained by removing all the side conditions of $(\mathrm{O} 6)$. Martin's book [10] defines a fundamental fold operation that is operation $(G)$ with the following finiteness condition:"If for two given points $P$ and $Q$ and for given lines $p$ and $q$ there are only a finite number of lines $t$ such that both $P^{t}$ is on $p$ and $Q^{t}$ is on $q$ ". Martin uses the notation $P^{t}$ to denote the reflection of point $P$. He further showed that some simpler operations (Yates postulates) can be derived from the fundamental fold operation. We extend this by showing that all in sold operations can be a achieved using $(G)$, in particular under what conditions a finite number of fold lines is achieved. We refine the above Martin's statement using the results obtained so far in this paper.

We will show how the degenerate and incident cases of $(\mathrm{G})$ realize the rest of the operations. We first consider the degenerate case of $(\mathrm{G})$, i.e. $m=n \wedge P=Q$. This case generates the infinite set of fold lines $\Gamma(P, m)$. Furthermore, when the arguments of $(\mathrm{O} 6)$ are more constrained, $(\mathrm{O} 6)$ is reduced to $(\mathrm{O} 2)$ and $(\mathrm{O} 3)$. Suppose $(\mathrm{O} 6)$ is given two s-pairs $(P, m)$ and $(Q, n)$, and further that $P \in$ $n \wedge Q \in m$, we have Lemmas 1 and 2 below. In the following, we denote by $\{\mathrm{O} i\}$, $i=1, \ldots, 7$, the set of fold lines that operation $(\mathrm{O} i)$ can generate.

315 Lemma 1. $\forall$ s-pairs $(P, m)$ and $(Q, n)$ that are origami constructible, if $m \neq$ $n \wedge P=Q \wedge(P \in n \wedge Q \in m)$ then $\{\mathrm{O} 6\} \subseteq\{\mathrm{O} 3\}$.

Proof. (Sketch) To perform (O6), $P$ and $Q$ have to be the intersection of $m$ and $n$. (O6) then generates the two bisectors of the angle formed by $m$ and $n$. Those lines are constructible by (O3) using $m$ and $n$.

Lemma 2. $\forall$ s-pairs $(P, m)$ and $(Q, n)$ that are origami constructible and satisfy $(P \notin m \wedge Q \notin n)$, if $m \neq n \wedge P \neq Q \wedge(P \in n \wedge Q \in m)$, then $\{\mathrm{O} 6\} \subseteq\{\mathrm{O} 2\} \cup\{\mathrm{O} 3\}$.

Proof. (Sketch) Under the condition $m \neq n \wedge P \neq Q \wedge(P \in n \wedge Q \in m)$, (O6) generates three fold lines, i.e. $P \uparrow Q$ and the two bisectors of the angle formed by $m$ and $n$. The first one is constructible by (O2) (cf. Fig. 1(a)), and the latter ones by (O3) (cf. Fig. 1(b)).

Theorem 1. $\forall$ s-pairs $(P, m)$ and $(Q, n)$ that are origami constructible,

$$
\begin{gathered}
\neg((P \in m \wedge Q \in n \wedge(m \| n \vee P=Q)) \vee \\
(P \notin m \wedge Q \notin n \wedge m=n \wedge P=Q)) \Rightarrow \\
\{\mathrm{G}\}=\bigcup_{i=1, \ldots, 7}\{\mathrm{Oi}\} .
\end{gathered}
$$




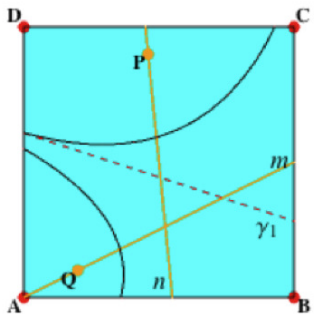

(a) (O2) performed by (G)

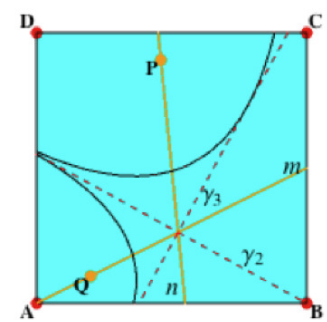

(b) (O3) performed by (G)

Fig. 1. (O2) and (O3) by (G) when $m \neq n \wedge P \neq Q$

Proof. We first prove that $(\mathrm{G})$ is reduced to (O1), (O4), (O5), (O6) and (O7) under certain configurations of the parameters. This implies that under such conditions, $\{\mathrm{G}\} \subseteq\{\mathrm{Oi}\}$, where $i=1,4,5,6$ and 7 .

We distinguish four cases.

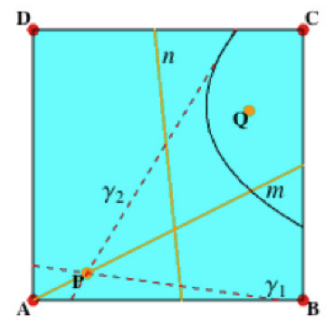

(a) (O5) performed by (G) when $P$ does not move

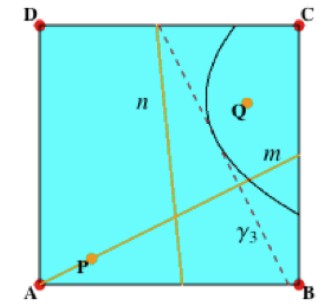

(b) (O7) performed by (G) when $P$ moves

Fig. 2. Incident case of $(\mathrm{G})$ for $P \in m \wedge Q \notin n$

1. $P \notin m \wedge Q \notin n$

If $(m=n \wedge P=Q)$, i.e. the degenerate case, then $(\mathrm{G})$ is an undefined (O6).

2. $P \in m \wedge Q \notin n$

We further distinguish two cases of fold; $P$ does not move, and $P$ moves along $m$. In the former case, the fold line passes through $P$ and superposes $Q$ and $n$, which is the case of (O5) as shown in Fig. 2(a). In the latter case, the fold line is a perpendicular to $m$ and superposes $Q$ and $n$, which is the case of (O7) (cf. Fig. 2(b)).

3. $P \notin m \wedge Q \in n$

Similarly to the case 1, we have the cases of (O5) and (O7). 
4. $P \in m \wedge Q \in n$

We further distinguish the following four cases:

(a) $m \| n$

The fold lines are perpendiculars common to the lines $m$ and $n$. They are infinite and form the set $\mathcal{B}(m)$.

(b) $\neg(m \| n) \wedge(P=Q)$

This is the case that $P$ is the intersection of $m$ and $n$. Any line passing through $P$ is the fold line. Therefore, we have the set of infinite number of fold lines $\mathcal{I}(P)$. In this case neither $P$ or $Q$ does move by the fold.

(c) $\neg(m \| n) \wedge(P \neq Q)$

We distinguish the following three cases:

i. $P$ moves and $Q$ does not move

The fold line is the perpendicular to $m$ passing through $Q$ that superposes $P$ and $m$. This is the case of (O4) (cf. Fig. 3(a)).

ii. $Q$ moves and $P$ does not move.

Similarly to the above case, we have the case of $(\mathrm{O} 4)$.

iii. Neither $P$ or $Q$ moves.

The fold line is $P Q$ constructible by (O1) (cf. Fig. 3(b)).

Table 3 summarizes the relations of $(\mathrm{G})$ and corresponding operations of $\mathbf{H}$ for all possible combinations of the conditions.

The condition to eliminate the infinite cases are as follows.

$$
\begin{array}{r}
(P \in m \wedge Q \in n \wedge(m \| n \vee P=Q)) \vee \\
(P \notin m \wedge Q \notin n \wedge m=n \wedge P=Q)
\end{array}
$$

Furthermore, by Lemmas 1 and 2, (O6) can be reduced to (O2) and (O3) under certain conditions. Therefore we obtain the following result

$$
\begin{aligned}
& \neg((P \in m \wedge Q \in n \wedge(m \| n \vee P=Q)) \vee \\
& (P \notin m \wedge Q \notin n \wedge m=n \wedge P=Q)) \Rightarrow \\
& \{\mathrm{G}\} \subseteq \bigcup_{i=1, \ldots, 7}\{\mathrm{Oi}\} .
\end{aligned}
$$

The relation

$$
\{\mathrm{G}\} \supseteq \bigcup_{i=1, \ldots, 7}\{\mathrm{Oi}\}
$$

can be shown as follows. For each $(\mathrm{O} i)$ we add parameters that satisfy the constraints to $(\mathrm{O} 6)$ operation as shown in Table 3 in the case of $(\mathrm{O} 1),(\mathrm{O} 4)$ - (O7) and the conditions stated in Lemmas 1 and 2 in the case of $(\mathrm{O} 2)$ and (O3).

Theorem 1 states that the principle $\mathbf{G}$ is as good as $\mathbf{H}$, although $\mathbf{G}$ is much simpler under the condition

$$
\begin{array}{r}
\neg((P \in m \wedge Q \in n \wedge(m \| n \vee P=Q)) \vee \\
(P \notin m \wedge Q \notin n \wedge m=n \wedge P=Q)) .
\end{array}
$$


Table 3. (G) to perform (O1), (O4) - (O7)

\begin{tabular}{|l|c|c|c|}
\hline incidence & degeneracy & operation & movement \\
\hline$P \in m, Q \in n$ & $m \| n$ & $\mathcal{B}(m)$ & $(\leftrightarrow, \leftrightarrow)$ \\
& $\neg(m \| n) \wedge P=Q$ & $\mathcal{I}(P)$ & $(\cdot, \cdot)$ \\
& $\neg(m \| n) \wedge P \neq Q$ & $(\mathrm{O} 1)$ & $(\cdot, \cdot)$ \\
& $\neg(m \| n) \wedge P \neq Q$ & $(\mathrm{O} 4)$ & $(\leftrightarrow, \cdot)$ \\
& $\neg(m \| n) \wedge P \neq Q$ & $(\mathrm{O} 4)$ & $(\cdot, \leftrightarrow)$ \\
\hline$P \in m, Q \notin n$ & & $(\mathrm{O} 5)$ & $(\cdot, *)$ \\
& & $(\mathrm{O} 7)$ & $(\leftrightarrow, *)$ \\
\hline$P \notin m, Q \in n$ & & $(\mathrm{O} 5)$ & $(*, \cdot)$ \\
& & $(\mathrm{O} 7)$ & $(*, \leftrightarrow)$ \\
\hline$P \notin m, Q \notin n$ & $m=n \wedge P=Q$ & $\Gamma(P, m)$ & $(*, *)$ \\
& $\neg(m=n \wedge P=Q)$ & $(\mathrm{O} 6)$ & $(*, *)$ \\
\hline
\end{tabular}

Note:

- Expression $(x, y)$ denotes the movements $x$ and $y$ of points $P$ and $Q$, respectively.

- We denote movement $x$ (or $y$ ) by symbols: "move" by " $\leftrightarrow$ ", "non-move" by "." and "do-not-care" by "*".

So let us define $\mathbf{G}^{\prime}$ as $\mathbf{G}$ with the above condition. Nevertheless, $\mathbf{G}^{\prime}$ has the following drawback. $\mathbf{G}^{\prime}$ may create lines whose geometrical properties are different. During origami construction, a fold step may give rise to multiple possible fold lines. The user should choose a suitable fold line among the possible ones. However, in proving geometrical properties by algebraic methods like Gröbner bases, this is likely to cause problems, since the property that we want to prove may be true only for certain choices. For example, when $P \in m$ and $Q \in n, \mathbf{G}^{\prime}$ generates two kinds of fold lines whose geometrical meaning are different, namely those by $(\mathrm{O} 4)$ and $(\mathrm{O} 1)$. In Fig. 3(a), the fold line $\gamma_{1}$ is perpendicular to $m$, whereas in Fig. 3(b), $\gamma_{2}$ is not necessary perpendicular to $m$. Although, the user chooses either $\gamma_{1}$ or $\gamma_{2}$ to perform the construction, the proof by Gröbner bases includes both cases. If the property that we want to prove depends on the perpendicularity of the fold line and line $m$, then the proof fails since perpendicularity doesn't hold for $\gamma_{2}$.

\section{Fold with Conic Sections}

We further explore the possibility of strengthening the power of origami. We extend Huzita's basic operations to allow solving polynomial equations of certain degrees while maintaining the manipulability of origami by hand. It has been shown in [9] that an extension that combines the use of compass with origami leads to interesting origami constructions, but does not increase the construction power of origami beyond what is constructible by $\mathbf{H}$. The extension generates 


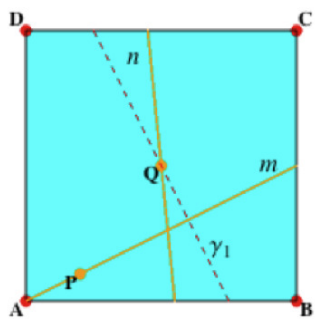

(a) (O4) performed by (G) when $P$ moves and $Q$ does not move

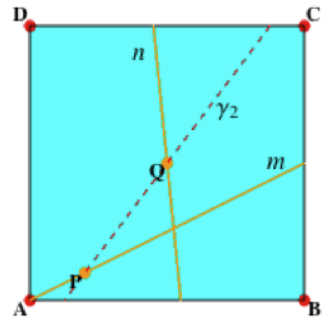

(b) (O1) performed by (G) when neither $P$ nor $Q$ moves

Fig. 3. Incident case of (G) for $P \in m \wedge Q \in n$

polynomial equations of degree 4 , which can be reduced to equations of degree 3.

It is also possible to increase origami power by allowing multi-fold as suggested by Alperin and Lang [2]. Although the $m$-fold method generates an arbitrarily high degree polynomial, accurately folding origami by $m$-lines simultaneously would be difficult even for $m=2$.

We further explore the foldability involving superposition of points and more general curves, which are still constructible using simple tools. In this section, we study the superposition with conic sections and describe the algebraic properties of the fold operation that superposes one point and a line, and superposes another point and a conic section assumed to be on the origami. This operation is realizable by hand and furthermore we expect to have a finite number of fold lines, which ensures the foldability. We consider a fold operation that simultaneously superposes two points with two conic sections to be difficult to perform by hand. Besides, folding to superpose a point with a conic section, with other combinations of simultaneous superpositions involving points and lines can be reduced to a more general one: superposition of two points with a line and a conic section.

To illustrate folding with conic sections by hand, an ellipse, parabola and hyperbola can be drawn on origami using pins, strings, a pencil and a straightedge, where only origami constructible points and lengths are used. Abstracting from the method used to draw a particular conic section on origami, we state the following fold operation in general:

400 - Given two points $P$ and $Q$, a line $m$ and a conic section $\mathcal{C}$, where $P$ is not on $\mathcal{C}$ and $Q$ is not on $m$, fold $\mathcal{O}$ along a line to superpose $P$ and $m$, and $Q$ and $\mathcal{C}$.

With little modification of the analysis performed with (O6) in Section 5.2, we obtain the following result, which corresponds to Proposition 6 for (O6).

Proposition 8. Given origami constructible points $P$ at $(a, b)$ and $Q$ at $(c, d)$, an origami constructible line $m:=y=0$, and a conic section $\mathcal{C}:=A x^{2}+B x y+$ 
$C y^{2}+D x+E y+F=0$, where coefficients $A, B, \ldots, F$ are origami constructible numbers and not all $A, B$ and $C$ are zero. We assume that $P$ is not on $m$ and $Q$ is not on $\mathcal{C}$. Let $\gamma$ be the fold line to superpose $P$ and $m$, and $Q$ and $\mathcal{C}$. Then the slope $t$ of $\gamma$ satisfies the following polynomial equation of degree six in $t$.

$$
\begin{gathered}
b B c+A c^{2}+b^{2} C-B c d-2 b C d+C d^{2}+c D+b E-d E+F+ \\
\left(-b^{2} B-2 A b c-2 a B c+2 B c^{2}-4 a b C+4 b c C+3 b B d+\right. \\
\left.4 A c d+4 a C d-4 c C d-2 B d^{2}-b D+2 d D-2 a E+2 c E\right) t+ \\
\left(A b^{2}+4 a b B+4 a A c-4 b B c-2 A c^{2}+4 a^{2} C-2 b^{2} C-\right. \\
8 a c C+4 c^{2} C-4 A b d-6 a B d+6 B c d+4 b C d+4 A d^{2}- \\
\left.2 C d^{2}+2 a D+2 F\right) t^{2}+ \\
\left(-4 a A b-4 a^{2} B+2 b^{2} B+4 A b c+6 a B c-2 B c^{2}+4 a b C-\right. \\
4 b c C+8 a A d-4 b B d-4 A c d-4 a C d+4 c C d+2 B d^{2}+ \\
2 d D-2 a E+2 c E) t^{3}+ \\
\left(4 a^{2} A-2 A b^{2}-4 a b B-4 a A c+3 b B c+A c^{2}+b^{2} C+B c d-\right. \\
\left.4 A b d+2 a B d-2 b C d+C d^{2}+2 a D-c D-b E+d E+F\right) t^{4}+ \\
\left(4 a A b-b^{2} B-2 A b c+b B d+b D\right) t^{5}+A b^{2} t^{6}
\end{gathered}
$$

Proof. (Sketch) Let points $U$ and $V$ be the reflections of $P$ and $Q$ respectively across the fold line $\gamma$. Point $U$ is on line $m$ and point $V$ is on the given conic section $\mathcal{C}$. Furthermore, fold line $\gamma$ is the perpendicular bisector of segments $P U$ and $Q V$. From the equations of these relations, with algebraic manipulation by a computer algebra system, we can derive the above degree six equation in slope $t$ of line $\gamma$.

This equation looks laborious; one should only note that it is an equation in $t$ of degree six over the field of origami constructible numbers.

In the example shown in Fig. 4, we assume origami constructible points $P$ at $(3,-4), Q$ at $(-1,1)$ and $m:=y=0$. The conic section $\mathcal{C}:=2 x^{2}+2 x y+y^{2}+$ $x+2 y-10=0$ is an ellipse depicted in Fig. 4. Giving concrete numerical values that realize the figure, we obtain the following equation for $t$.

$$
16 t^{6}-78 t^{5}+84 t^{4}+39 t^{3}-66 t^{2}+t+8=0
$$

Solving Eq. (7.1) using Mathematica 8 yields six real solutions that correspond to six possible fold lines $k_{1}, \cdots, k_{6}$ in Fig. 4 . The same operation can be performed by hand, obtaining fold lines with certain slopes. Each slope value is one real solution to the equation.

\section{Concluding Remarks}

We reformulated the Huzita's operations giving them precise definitions with side conditions that eliminate the degenerate and incident cases. We showed that for each of the reformulated operations only a finite number of fold lines is possible. We gave an alternative single operation based on operation (O6) and showed 


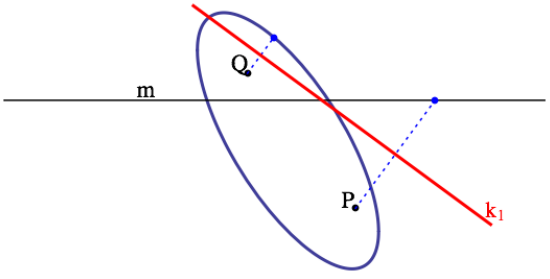

(a)

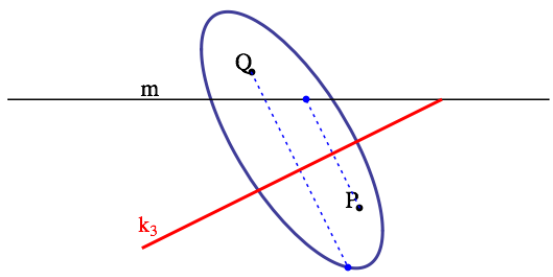

(c)

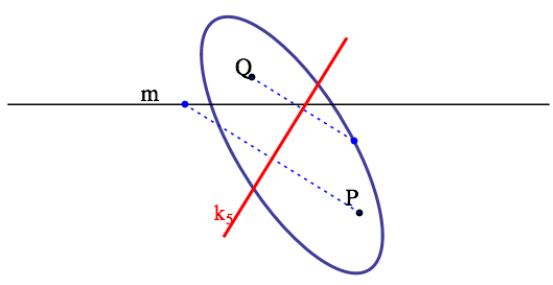

(e)

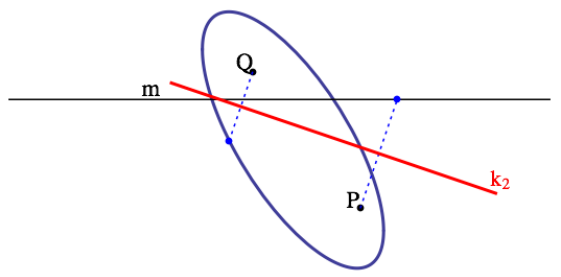

(b)

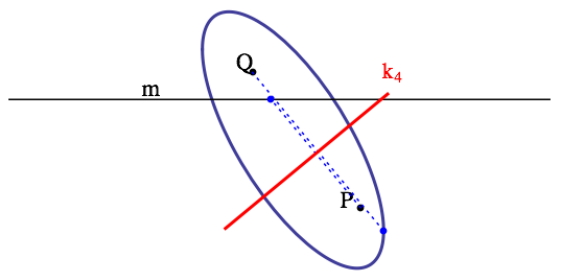

(d)

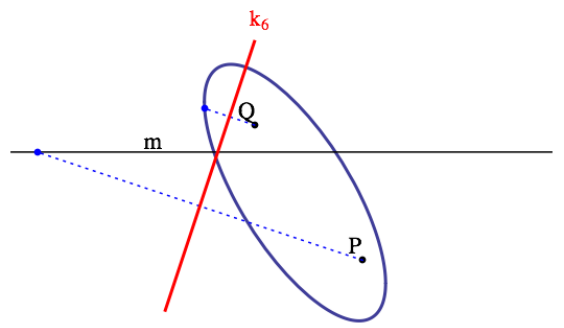

(f)

Fig. 4. Fold lines $k_{1}, \cdots, k_{6}$ whose slopes are the six distinct real solutions of the equation $16 t^{6}-78 t^{5}+84 t^{4}+39 t^{3}-66 t^{2}+t+8=0$

how each of the reformulated operations can be performed using the new one. Furthermore, we investigated the combination of origami operations and conic sections. We showed that finding a fold line that superposes two points, one with a line and the other with a conic section, is reduced to solving an equation of degree six. We can think of two directions for future work of this research.

First, the principles of folding presented in this paper have been worked out carefully, so that they can be formalized in a proof assistant. Starting from a formalization of the basic geometric concepts, one can formally define the lines (or sets of lines) that arise from particular fold operations. This can be used to specify the superpositions that arise from the composition of fold operations, and the set of origami constructible points and lines. We imagine that such a development would give a basis for a formalized origami theory. Recently, it has been shown [8], that the decision procedures already present in modern proof assistants combined with the symbolic computation procedures are strong enough to solve many of the goals arising in computational origami problems. 
Second, further investigation of fold operations involving conic sections is required to give exact definitions of the fold operations with their degenerate and incident cases. We showed that superposing two points onto line and conic section gives rise to equation of degree six. However the bigger question is whether this fold operation would solve all the equations of degree five and six. In other words, can we find an algorithm for translating degree five and degree six equations, possibly with certain conditions, into origami fold problems?

\section{References}

1. R. C. Alperin. A Mathematical Theory of Origami Constructions and Numbers. New York Journal of Mathematics, 6:119-133, 2000.

2. R. C. Alperin and R. J. Lang. One-, Two, and Multi-fold Origami Axioms. In Origami ${ }^{4}$ Fourth International Meeting of Origami Science, Mathematics and Education, pages 371-393. A K Peters Ltd, 2009.

3. F. Ghourabi, T. Ida, H. Takahashi, and A. Kasem. Reasoning Tool for Mathematical Origami Construction. In CD Proceedings of the International Symposium on Symbolic and Algebraic Computation (ISSAC 2009), 2009.

4. H. Huzita. Axiomatic Development of Origami Geometry. In Proceedings of the First International Meeting of Origami Science and Technology, pages 143-158, 1989.

5. T. Ida, A. Kasem, F. Ghourabi, and H. Takahashi. Morley's theorem revisited: Origami construction and automated proof. Journal of Symbolic Computation, 46(5):571 - 583, 2011.

6. A. Jones, S. A. Morris, and K. R. Pearson. Abstract Algebra and Famous Impossibilities. Springer-Verlag New York, Inc., 1991.

7. J. Justin. Résolution par le pliage de l'équation du troisième degré et applications géométriques. In Proceedings of the First International Meeting of Origami Science and Technology, pages 251-261, 1989.

8. C. Kaliszyk and T. Ida. Proof Assistant Decision Procedures for Formalizing Origami. In J. H. Davenport, W. M. Farmer, J. Urban, and F. Rabe, editors, Proc. of the 4th Conference on Intelligent Computer Mathematics (CICM'11), volume 6824 of $L N C S$, pages 45-57. Springer Verlag, 2011.

9. A. Kasem, F. Ghourabi, and T. Ida. Origami Axioms and Circle Extension. In Proceedings of the 26th Symposium on Applied Computing, pages 1106-1111. ACM press, 2011.

475 10. G. E. Martin. Geometric Constructions. Springer-Verlag New York, Inc., 1998.

11. P. L. Wantzel. Recherches sur les moyens de connaitre si un problème de géométrie peut se résoudre avec la règle et le compas. Journal de Mathématiques Pures et Appliquées, pages 366-372, 1984. 